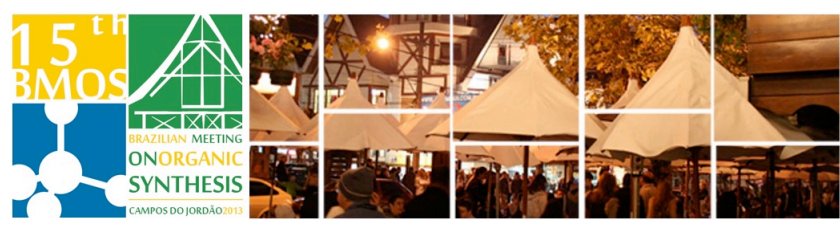

\title{
Synthesis and evaluation of phytotoxic activity of triazole derivatives of tris(hydroxymethyl)amino methane
}

\section{Felipe Purisco Gomes Euzébio (PG) ${ }^{1}$; Rosemeire Brondi Alves (PQ) ${ }^{1 *}$; Rossimiriam Pereira de Freitas (PQ) ${ }^{1}$}

\author{
${ }^{1}$ Departamento de Química, ICEx, Universidade Federal de Minas Gerais
}

*brondi@netuno.lcc.ufmg.br

Keywords: triazoles; phytotoxic activity; "click chemistry"

\section{INTRODUCTION}

The number of agrochemicals containing halogen has been increasing along the years. Modern agrochemicals have been obtained by introducing halogens into active ingredients and are developed seeking optimal efficacy, environmental safety, user friendliness and economic viability. ${ }^{1}$ In this context, triazoles containing halogenated aryl moieties were synthesized and their phytotoxic activities were evaluated.

\section{RESULTS AND DISCUSSION}

The triazoles were obtained via "click" reaction between the alkynes $(3 a-3 i)$ and the azide $6^{2}$, as shown in Scheme 1.

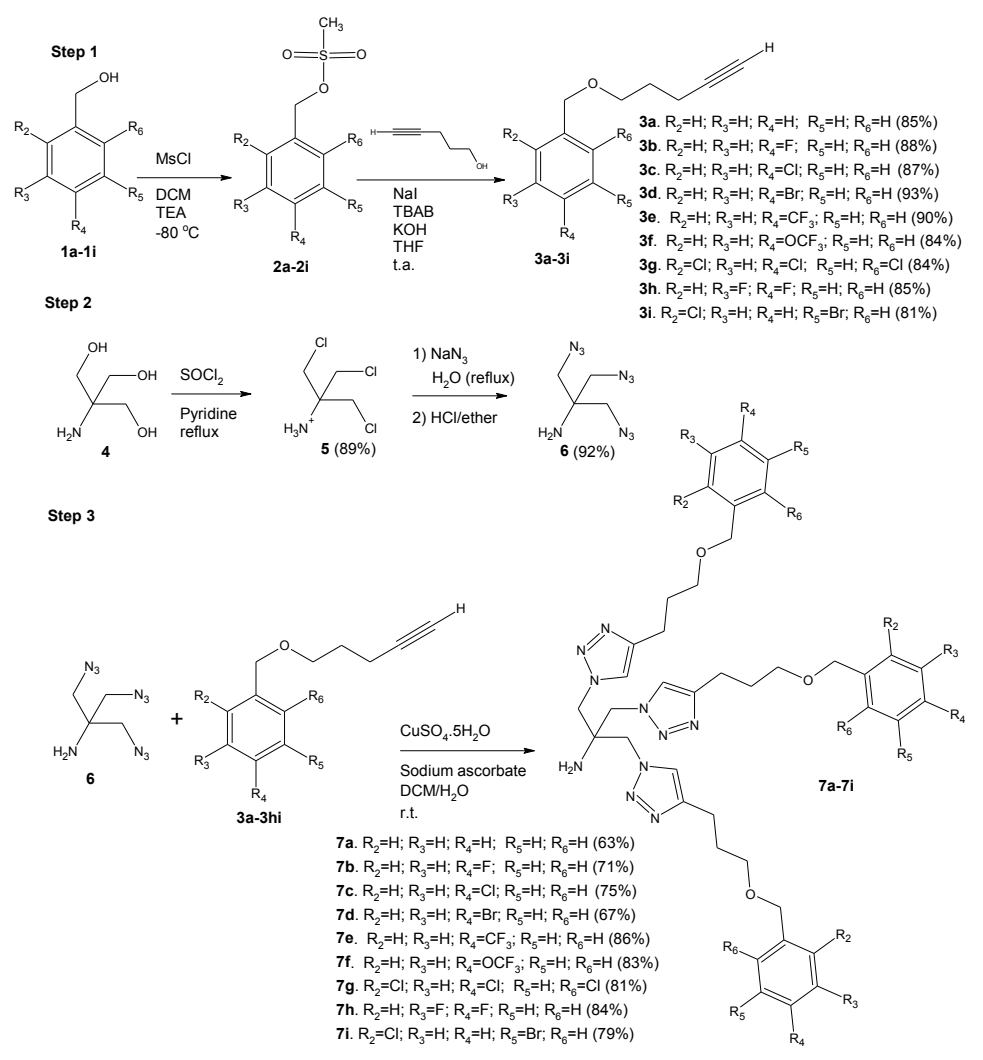

Scheme 1. Preparation of triazoles 7a-7i.
Tris(hydroxymethyl)amino methane (4) was initially converted into its corresponding chloride $(5)^{3}$ and then converted into azide $6^{4}$. Comercially benzyl alcohols (1a-1i) were initially converted into their corresponding mesylates $(\mathbf{2} \mathbf{a}-\mathbf{2} \mathbf{i})$ in yields ranging from $86 \%$ to $96 \%)^{5}$ and then converted into their corresponding ethers (3a-3i) in yields ranging from $81 \%$ to $93 \%{ }^{6}$. The triazoles' biological activities were evaluated with respect to the germination and radicle growth inhibition of three dicotyledonous species: Cucumis sativus, Lactuca sativa and Bidens pilosa ${ }^{7}$. Best results were observed for triazol 7f, which contains the halogenated group -OCF and $\mathbf{7 g}$, which contains three chloro atoms. In addition, the halogenated compounds had, in general, superior effects relative to that of their nonhalogenated counterpart (compound 7a), demonstrating the beneficial effects of the presence of halogen atoms on the biological activity of the evaluated compounds.

\section{CONCLUSION}

In summary, nine new 1,2,3-triazoles were synthesized, purified and fully characterized. The conditions employed were satisfactory, and thus, we did not observe the formation of side products. The comparison of the phytotoxic activities of products $\mathbf{7 b} \mathbf{b} \mathbf{i}$ with the activities of compound $\mathbf{7 a}$, which was synthesized to evaluate the influence of the absence of an halogen substituent on activity, revealed that the halogenated products were more active.

\section{ACKNOWLEDGEMENTS}

We are grateful to CNPq, FAPEMIG and PRPq/UFMG for their financial support.

\footnotetext{
REFERENCES

Jeschke, P. Pest Man. Sci., 2010, 66, 10.

${ }^{2}$ lehl, J.; De Freitas, R. P.; Nierengarten, J. F. Tetrahedron Let., 2008, 49, 4063.

${ }^{3}$ Martinu, T.; Dailey, W. P. J. Org. Chem., 2000, 65, 6784.

${ }^{4}$ Díaz, D. D., Punna, S., Holzer, P., Mcpherson, A. K., Sharpless, K. B. J. Pol. Sci. Part A, 2004, 42, 4392.

${ }^{5}$ Bundy, G. L.; Lin, C. H.; Sih, J. C. Tetrahedron, 1981, 37, 4419.

6 Kaplánek, R.; Bríza, T.; Havlík, M.; Martásek, P.; Král, V. J. Fluor. Chem. 2007, 128, 179.

7 Macías, F. A.; Castellano, D.; Molinillo J. M. G. J. Agric. Food Chem., 2000, 48, 2512.
} 